\title{
Validación y acreditación de procedimientos analíticos de macronutrientes: aplicación al análisis nutricional en purés de dietas geriátricas
}

\author{
Pérez Grana R.
}

Sanid. mil. 2015; 71 (4): 224-231; ISSN: 1887-8571

\section{RESUMEN}

Antecedentes y objetivos: Al objeto de asegurar la calidad en la determinación de macronutrientes de las conservas de raciones de combate del Ejército de Tierra y de platos cocinados, se realiza la validación y acreditación de los procedimientos analíticos, y se aplican al análisis nutricional de purés, pertenecientes a dietas geriátricas de una Residencia Militar de Atención a Mayores de la Dirección de Asistencia al Personal del Ejército de Tierra. Material y métodos: Los ensayos se realizan con procedimientos analíticos internos. La estimación de la incertidumbre se realiza según los principios de la guía EURACHEM/CITAC. Resultados: Rango de valores en ensayos de materiales de referencia certificados (MRC): HUMEDAD: sesgo relativo, 0,19-3,86\%; DER $\mathrm{R}_{\mathrm{R}} 0,31-1,76 \%$; $\mathrm{DER}_{\mathrm{r}} 0,25-0,86 \% ; \mathrm{HORRAT}_{\mathrm{r}} 0,15-0,72 ; \mathrm{U}_{\text {rel }}$

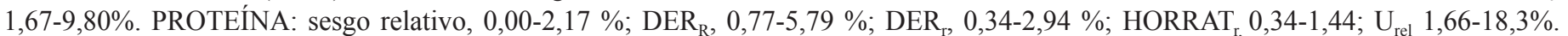
Límite de detección (LD), 0,08\% de nitrógeno. Límite de cuantificación (LC), 0,29 \% de nitrógeno. CENIZAS: sesgo relativo, 2,50-13,63\%; $\mathrm{DER}_{\mathrm{R}}, 2,42-16,62 \%$; $\mathrm{DER}_{\mathrm{r}}, 0,62-8,26 \%$; $\mathrm{HORRAT}_{\mathrm{r}}$ 0,65-3,30; $\mathrm{U}_{\text {rel }}$ 6,86-45,7\%. GRASA: sesgo relativo, 3,74-5,88\%; DER $\mathrm{R}$ 0,98-3,32\%; DER $_{\mathrm{r}}$ 0,94-3,22\%; HORRAT $\mathrm{r}$ 0,34-1,36\%; $\mathrm{U}_{\text {rel }} 13,0-15,8 \%$. En los ensayos de aptitud se obtiene un Z-score $\mathrm{z} \leq 2$. Las 51 muestras de purés, elaborados de forma culinaria, arrojan los siguientes valores medios, en porcentaje: humedad $(81,3 \pm 4,0)$; proteína $(5,17 \pm 2,14)$; grasa $(3,15 \pm 1,27)$; cenizas $(0,98 \pm 0,48)$. Conclusiones: Los procedimientos analíticos son útiles en el rango de determinación. El alcance de la acreditación (ENAC, España) afecta a humedad (40-95\%); proteína (0,5-46\%), grasa (3,5-50\%) y cenizas $(0,4-4 \%)$.

PALABRAS CLAVE. Validación. Acreditación. Macronutrientes. Conservas. Raciones de combate. Purés. Dietas geriátricas.

Validation and accreditation of analytical procedures of macronutrients: application to nutritional analysis in purées of geriatric diets SUMMARY: Backgrounds and objectives: In order to ensure the quality in determining macronutrients in canned foods of combat rations Spanish Army and cooked dishes, validation and accreditation of analytical procedures are carried out. These are applied to nutritional analysis in purées of geriatric diets of a Military Old People's Home of the Direction of Personal Assistance Spanish Army. Material and Methods: Assays were performed with internal analytical procedures. The uncertainty estimation is performed according to the principles of EURACHEM/CITAC guide. Results: Value range of assays of certified reference materials (CRMs): MOISTURE: relative bias, 0,19-

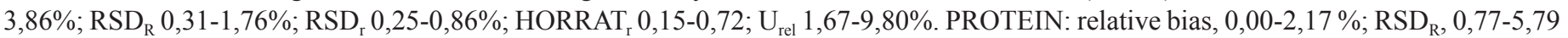
$\%$; $\mathrm{RSD}_{\mathrm{r}}, 0,34-2,94 \%$; HORRAT $, 0,34-1,44 ; \mathrm{U}_{\text {rel }}$ 1,66-18,3\%. Limit of detection (LOD), 0,08\% of nitrogen. Limit of quantitation (LOQ),

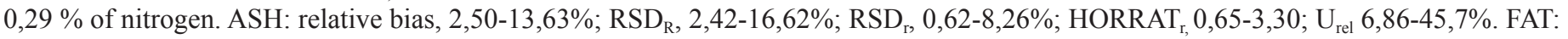
relative bias, 3,74-5,88\%; $\mathrm{RSD}_{\mathrm{R}}$ 0,98-3,32\%; RSD $0,94-3,22 \%$; HORRAT $_{\mathrm{r}}$ 0,34-1,36\%; $\mathrm{U}_{\text {rel }}$ 13,0-15,8\%. Proficiency testing exercices got a Z-score $\mathrm{z} \leq 2$. The analysis of 51 purées samples, elaborated in a culinary form, yield the following mean values in percent: moisture $(81,3 \pm 4,0)$; protein $(5,17 \pm 2,14)$; fat $(3,15 \pm 1,27)$; ash $(0,98 \pm 0,48)$. Conclusions: The analytical procedures are useful within the range of determination. The reach of accreditation (ENAC, Spain) affects moisture (40-95\%); protein (0,5-46\%), fat (3,5-50\%) and ash (0,4-4\%).

KEYWORDS. Validation. Accreditation. Macronutrients. Canned foods. Combat rations. Purées. Geriatric diets.

\section{INTRODUCCIÓN}

Las personas en edad geriátrica presentan mayor frecuencia y riesgo de deficiencias nutricionales, aunque en muchas ocasiones de forma moderada. Suelen ser debidas a una mala planificación y adaptación de los menús ofrecidos que no llegan a cubrir las nece-

${ }^{1}$ Tcol. Veterinario. Centro Militar de Veterinaria de la Defensa. Servicio de Bromatología y Seguridad Alimentaria. Madrid. España.

Dirección para correspondencia: Roberto Pérez Grana. Servicio de Bromatología y Seguridad Alimentaria. Centro Militar de Veterinaria de la Defensa. C/ Darío Gazapo, 3 28024. Madrid. Tfno.915122515.rpergra@oc.mde.es; rperezgrana@hotmail.com

Recibido: 19 de febrero de 2015

Aceptado: 9 de abril de 2015 sidades recomendadas de energía, macronutrientes y micronutrientes $^{1}$. En este sentido la Ley de Seguridad Alimentaria ${ }^{2}$ y Nutrición, establece que se asegurará una correcta alimentación en todas las etapas de la vida y se prestará especial interés a los problemas de desnutrición.

La malnutrición puede afectar al $59 \%$ de los ancianos que viven residencias $^{3}$, llegando incluso hasta al $85 \%$ en el caso de ancianos institucionalizados mayores de 75 años. La malnutrición calcioproteica y el déficit de vitaminas ( $\mathrm{A}, \mathrm{B}_{6}, \mathrm{~B}_{12}, \mathrm{C}, \mathrm{D}$, ácido fólico), calcio y zinc son los más frecuentes ${ }^{4}$. Por consiguiente, las guías de nutrición aconsejan incrementar el aporte de proteínas hasta el $1-1,1 \mathrm{~g} / \mathrm{kg} /$ día, frente a los $0,8 \mathrm{~g} / \mathrm{kg} /$ día que se recomiendan para la población adulta ${ }^{5}$.

El análisis nutricional permite conocer de una forma exacta el contenido real de nutrientes en los alimentos. Con las tablas de com- 
posición de alimentos, tan solo se consigue una información semicuantitativa, más exacta, para los macronutrientes o algunos constituyentes inorgánicos ${ }^{6}$.

Los procedimientos analíticos o de ensayo de macronutrientes (humedad, proteína, grasa y cenizas) para el análisis nutricional en conservas de raciones de combate del Ejército de Tierra y platos cocinados, fueron acreditados por ENAC (Entidad Nacional de Acreditación, España), conforme a la norma ISO 17025.

La norma ISO $8402^{7}$ define la validación como la confirmación mediante examen y provisión de evidencias objetivas de que se cumplen los requisitos particulares para un uso específico determinado.

La norma ISO/IEC $17025^{8}$ indica que los resultados analíticos deben de ir acompañados de su incertidumbre, parámetro más importante que describe la calidad de una medida, y afecta fundamentalmente a las decisiones que están basadas sobre el resultado de una medida.

La norma ISO 3534-99 define la incertidumbre como una estimación unida al resultado de un ensayo que caracteriza el intervalo de valores dentro de los cuales se afirma que se encuentra el valor verdadero. El JCGM (Joint Committee for Guides in Metrology) ${ }^{10}$ define la incertidumbre como un parámetro asociado con el resultado de una medida, que caracteriza la dispersión de valores que podrían razonablemente ser atribuidos al mesurando. El Vocabulario Internacional de Metrología (VIM) ${ }^{11}$ también define la incertidumbre como un parámetro, pero no-negativo, que caracteriza la dispersión de valores cuantitativos atribuidos a un mensurando.

La trazabilidad de los resultados analíticos se logra con el uso de materiales de referencia certificados (MRC), la calibración de equipos y mediante la validación del proceso analítico. El uso de MRC es suficiente para poder tener el grado suficiente de comparabilidad entre resultados sin tener que llegar a la unidad del Sistema Internacional de Unidades (SI).

La adecuación a la finalidad o requerimientos perseguidos "Fitness for purpose" es lo que debería llegar a declarar el método de validación. Este término es definido por la IUPAC ${ }^{12}$ como el nivel alcanzado por los datos producidos por un proceso de medida que permite al usuario tomar decisiones técnica y administrativamente correctas para un proceso determinado.

Por su parte, los estudios interlaboratorio complementan el proceso de validación, y se restringen a la precisión y a la determinación de la veracidad.

\section{MATERIAL Y MÉTODOS}

\section{Preparación de la muestra}

En el laboratorio se realiza la preparación física de las muestras de purés con una trituradora (Vorwek, Germany), hasta obtener la muestra analítica. La temperatura de la homogeneización no debe sobrepasar $24^{\circ} \mathrm{C}$. El tamaño de partícula $(\leq 1 \mathrm{~mm})$ se comprueba con un tamiz ISO 3310-1:2000, a partir de cinco alícuotas tomadas aleatoriamente.

\section{Procedimientos analíticos de macronutrientes}

Se realiza la validación y acreditación de los procedimientos analíticos de macronutrientes, y se aplican al análisis nutricional de 51 muestras de purés, elaborados de forma culinaria, pertenecientes a dietas geriátricas de una Residencia Militar de Atención a Mayores de la Dirección de Asistencia al Personal del Ejército de Tierra. Los citados procedimientos están basados en las normas ISO $1442^{13}$, ISO $937^{14}$, ISO $1443^{15}$, ISO $936^{16}$ y AOAC AO-19 $^{17} 950.46$; 979.09; 920.87; 920.153; 900.02 A ó B).

\section{Humedad}

Se pesan 5-5,5 $\mathrm{g} \pm 0,1 \mathrm{mg}$, en una balanza analítica (Sartorius, Barcelona), de la muestra analítica homogeneizada, en la cápsula de porcelana. La eliminación del agua se realiza en una estufa de desecación (Selecta, Barcelona) a $103{ }^{\circ} \mathrm{C} \pm 2{ }^{\circ} \mathrm{C}$ hasta obtener un peso constante. Rango de determinación: $40-95 \%$.

\section{Proteína}

Se pesa la muestra, según los porcentajes de proteína estimados que se espera que contenga la muestra real $(0-7 \%: 1-1,2 \mathrm{~g}$;

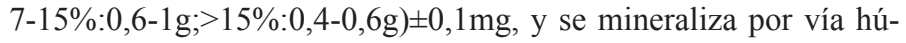
meda (Speed Digestor K-436, Büchi, Switzerland) con $15 \mathrm{ml}$ ácido sulfúrico concentrado, utilizando un catalizador $\left(1,5 \% \mathrm{SO}_{4} \mathrm{~K}_{2} .5 \mathrm{H}_{2} \mathrm{O}\right.$; $2 \% \mathrm{Se}$ ). Se añaden $75 \mathrm{ml}$ de una solución de hidróxido de sodio al $32 \%$ p/v, y el amoniaco liberado es arrastrado por destilación (Distilation Unit B-324, Büchi, Switzerland) y se recoge en ácido bórico al $4 \%$, y se titula con ácido clorhídrico $0,1 \mathrm{~N}$. La determinación de proteína comprende varias etapas:

$$
\underset{\mathrm{n}}{\text { 1. Digestión }} \underset{\text { proteina }}{\mathrm{C}-\mathrm{NH}_{2}}+\mathrm{mH}_{2} \mathrm{SO}_{4} \underset{\text { calor }}{\stackrel{\text { catalizadores }}{\longrightarrow}} \mathrm{CO}_{2}+\left(\mathrm{NH}_{4}\right)_{2} \mathrm{SO}_{4}+\mathrm{SO}_{2}
$$

2. Neutralización y destilación

$$
\begin{aligned}
& \left(\mathrm{NH}_{4}\right)_{2} \mathrm{SO}_{4}+2 \mathrm{NaOH} \longrightarrow \mathrm{NH}_{3}+\mathrm{Na}_{2} \mathrm{SO}_{4}+2 \mathrm{H}_{2} \mathrm{O} \\
& \mathrm{NH}_{3}+\mathrm{H}_{3} \mathrm{BO}_{3} \longrightarrow \mathrm{NH}_{4}+\mathrm{H}_{2}-\mathrm{BO}_{3} \text { (color verde) }
\end{aligned}
$$

\section{Titulación}

El anión borato (proporcional a la cantidad de nitrógeno) se titula con $\mathrm{HCl} 0,1 \mathrm{~N}$ estandarizado:

$\mathrm{H}_{2}-\mathrm{BO}_{3}+\mathrm{H}^{+} \longrightarrow \mathrm{H}_{3} \mathrm{BO}_{3}$ (color rojo)

El porcentaje de proteína (g/100 g de muestra) se obtiene multiplicando el porcentaje de nitrógeno hallado por 6,25. Rango de determinación: 0,5-46\%.

\section{Grasa}

Se pesa la muestra según los porcentajes de grasa estimados que se espera que contenga la muestra real $(0-10 \%: 1,5-2 \mathrm{~g} ; 10-20 \%$ : $1-1,5 \mathrm{~g} ;>20 \%: 0,8-1) \pm 0,1 \mathrm{mg}$, en una cápsula de vidrio con filtro. Se digiere la muestra (Soxcap 2047 FOSS, Sweden) con ácido clorhídrico 3-5N y se extrae la grasa (Soxtec System HT 1043, Tecator, Sweden) con éter de petróleo $40-60^{\circ} \mathrm{C}$, se deseca el residuo y se pesa. Rango de determinación: 3,5-50\%. 


\section{R. Pérez Grana}

\section{Cenizas}

Se pesan 5-5,5 g士0,1mg de la muestra analítica en un crisol de porcelana, se carboniza y se incinera en la mufla (Heron, Barcelona) a $550{ }^{\circ} \mathrm{C} \pm 10^{\circ} \mathrm{C}$, y el residuo de materia inorgánica se pesa. Rango de determinación: $0,4-4 \%$.

\section{Validación de procedimientos analíticos de macronutrientes}

Mediante un diseño experimental anidado ${ }^{20,21}$ se varían los factores analista, instrumento y día, de forma ordenada. Para evaluar la exactitud/sesgo y la precisión, se utilizan los siguientes MRC: humedad (LGC 7107 25,9 $\pm 1,2$ g/100g; LGC ERM-BB 501 a, 61,8 $\pm 0,7$ g/100; LGC 7105 71,3 $\pm 1,0$ g/100g), proteína (LGC Rice pudding $0,17 \pm 0,02 \mathrm{~g}$ de nitrógeno/100g; LGC ERM-BB $501 \mathrm{a}, 23,0 \pm 0,7 \mathrm{~g}$ de nitrógeno/Kg; MUVA-HA 46,70 $\pm 0,17 \mathrm{~g}$ de proteína/100g); gra-

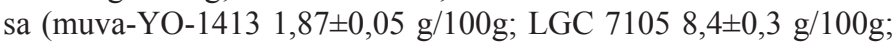
muva-HA-1508 27,52 $\pm 0,14 \mathrm{~g} / 100 \mathrm{~g}$ ) y cenizas (LGC Rice pudding 0,22 $\pm 0,05 \mathrm{~g} / 100 \mathrm{~g}$; LGC 7103 Sweet digestive biscuit 1,599 $\pm 0,077$

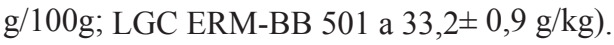

Además de los parámetros de la calidad, exactitud (propiedad analítica suprema) y precisión (propiedad analítica básica), en proteínas se determina el límite de detección (LD) y el límite de cuantificación (LQ). Para verificar la recuperación de nitrógeno se utilizan sustancias estándar como acetanilida (nitrógeno teórico, 10,36\%; Panreac, Barcelona) y glicina (nitrógeno teórico, 18,66\%, Fluka, Barcelona). Asimismo, se realizan ensayos de recuperación de grasa con matrices fortificadas (paté, caballa [Scomber scombrus] en aceite, melocotón [Prunus pérsica] en almíbar) con una cantidad conocida de aceite de ricino (pureza $\geq 99,7 \%$; Sigma-Aldrich, Madrid).

\section{Precisión}

Se estima la precisión de la repetibilidad $\left(\mathrm{DER}_{\mathrm{r}}\right)$ y la precisión de la reproductibilidad intralaboratorio $\left(\mathrm{DER}_{\mathrm{R}}\right)$. El límite de repetibilidad y el límite de reproductibilidad se calculan para un intervalo de confianza del $95 \%{ }^{22}$.

\section{Sesgo}

La estimación del sesgo se realiza comparando el valor medio obtenido al analizar los MRC con su valor certificado. Para demostrar si el sesgo es estadísticamente significativo y, en consecuencia, verificar la trazabilidad del procedimiento analítico, se usa el estadístico t, de dos colas, para determinar si el valor medio, es significativamente diferente del valor certificado, según la expresión:

$$
\mathrm{t}_{\text {exp }} \mathrm{t}_{\exp }=\frac{\left|X_{m}-\mu_{o}\right| \sqrt{n}}{S}
$$

Donde, $\mu_{\mathrm{o}}$ es el valor nominal del MRC, $\mathrm{X}_{\mathrm{m}}$ es el valor medio de los análisis del material de referencia certificado, $S$ es la desviación estándar de los resultados y $n$, es el número de medidas replicadas usadas para obtener el valor medio.

\section{Límite de detección y límite de cuantificación}

Se estiman a partir de 20 replicados de muestras blanco, de acuerdo con las ecuaciones: $\mathrm{LD}=\mathrm{X}_{\mathrm{b}}+3 \mathrm{~S}_{\mathrm{b}} ; \mathrm{LC}=\mathrm{X}_{\mathrm{b}}+10 \mathrm{~S}_{\mathrm{b}}$. Se expre- san en fracción de masa de nitrógeno. Siendo, $X_{b}$, el valor medio de los blancos, y $\mathrm{S}_{\mathrm{b}}$, la desviación estándar de los blancos.

\section{Incertidumbre}

Aplicando la ley de propagación de la incertidumbre tenemos la expresión básica que estima la incertidumbre:

$$
\mathrm{u}\left[\mathrm{y}\left(\mathrm{x}_{1}, \mathrm{x}_{2}, \ldots\right)\right]=\sqrt{\sum_{i=1, n} c_{i}^{2} u\left(x_{i}\right)^{2}}
$$

Donde $\mathrm{y}\left(\mathrm{x}_{1}, \mathrm{x}_{2}, \ldots . \mathrm{x}_{\mathrm{n}}\right)$ es una función de varias variables independientes $\mathrm{x}_{1}, \mathrm{x}_{2}, \ldots, \mathrm{y} \mathrm{c}_{\mathrm{i}}$ es el coeficiente de sensibilidad evaluado como $c_{\mathrm{i}}=\partial y / \partial x_{\mathrm{i}}$, que es la derivada parcial de y con respecto a $\mathrm{x}_{\mathrm{i}} ; \mathrm{u}$ $\left(\mathrm{x}_{\mathrm{i}}\right)$ y u (y) son la incertidumbre estándar y la incertidumbre estándar combinada.

La estimación de la incertidumbre combinada, $u_{c}$, se lleva a cabo agrupando términos, referentes a incertidumbre de MRC (incertidumbre de tipo B), precisión y sesgo. La incertidumbre expandida, $\mathrm{U}$, se obtiene aplicando un factor $\mathrm{k}=2$ a la incertidumbre combinada

\section{RESULTADOS}

En la tabla 1 se exponen los valores de los parámetros de la calidad de los procedimientos analíticos de humedad, proteína, grasa y cenizas. En proteínas, el LD y el LC, expresados en \% de nitrógeno, alcanzan valores del 0,08\% y el 0,29\%, respectivamente. Asimismo, se obtiene una recuperación de nitrógeno de $1,00 \pm 0,0033$ para glicina y acetanilida. Y en el caso de la glicina, se obtiene un valor medio en 20 determinaciones, y en diferentes días, del 18,66\% (expresado en nitrógeno) $\pm 0,30 \%$, en cuyo intervalo se considera que el método está bajo control estadístico.

En la figura 1 se presenta la curva de regresión lineal (grasa añadida versus grasa recuperada), el coeficiente de determinación $\left(\mathrm{R}^{2}=0,9942\right)$ y la ecuación de la recta. Se obtiene un valor de la recuperación media de grasa de $1,00 \pm 0,06$ y una $D_{E R}$ del 5,64\%.

En la tabla 2 se exponen las diferencias máximas a diferentes concentraciones en los parámetros estudiados, expresados en porcentaje, en condiciones de repetibilidad.

En la tabla 3 se exponen los resultados de los ensayos de aptitud $\mathrm{y}$, en la tabla 4 , se refleja la media aritmética, el valor máximo y mínimo en las 51 muestras de purés, y el análisis nutricional en 5 de ellas, consideradas representativas.

\section{DISCUSIÓN}

\section{Incertidumbre y muestreo}

Es interesante señalar que la ISO 17025 indica que, si el laboratorio no realiza ningún muestreo, únicamente debe informar de la incertidumbre del análisis. Por consiguiente, en los valores de incertidumbre de macronutrientes no está incluida la incertidumbre del muestreo.

La heterogeneidad de las muestras sólidas ${ }^{23}$ de alimentos supone una parte importante de la incertidumbre del muestreo, y puede variar ampliamente junto con la concentración de analito; por ello, se realiza un paso de homogeneización antes del ensayo. 
Validación y acreditación de procedimientos analíticos de macronutrientes: aplicación al análisis...

Tabla 1. Resultados de los parámetros de la calidad.

\begin{tabular}{|c|c|c|c|c|c|c|c|c|}
\hline MCR & $\mathbf{n}$ & $\begin{array}{c}M \\
\mathrm{~g} / \mathbf{1 0 0 g}\end{array}$ & $\begin{array}{c}\text { DER }_{\mathrm{R}} \\
\%\end{array}$ & $\begin{array}{c}\text { DER }_{\mathrm{r}} \\
\%\end{array}$ & $\begin{array}{l}\text { SA } \\
\%\end{array}$ & $\begin{array}{l}\text { SR } \\
\%\end{array}$ & HORRAT $_{r}$ & $\begin{array}{c}\mathrm{U}_{\text {rel, }} \\
\%\end{array}$ \\
\hline \multicolumn{9}{|l|}{ Humedad } \\
\hline LGC7107 & 12 & $26,9 \pm 0,3$ & 1,76 & 0,86 & 1,00 & 3,86 & 0,72 & 9,80 \\
\hline ERM BB 501 A & 12 & $61,7 \pm 0,3$ & 0,75 & 0,34 & 0,12 & 0,19 & 0,35 & 1,97 \\
\hline LGC 7105 & 12 & $71,0 \pm 0,1$ & 0,31 & 0,25 & 0,30 & 0,42 & 0,15 & 1,67 \\
\hline \multicolumn{9}{|l|}{ Grasa } \\
\hline muva-YO-1413 & 12 & $1,77 \pm 0,09$ & 2,68 & 2,65 & 0,07 & 3,74 & 0,73 & 13,3 \\
\hline LGC 7105 & 17 & $7,76 \pm 0,23$ & 0,98 & 0,94 & 0,64 & 7,61 & 0,34 & 15,8 \\
\hline muva-HA-1508 & 15 & $25,9 \pm 2,6$ & 3,32 & 3,22 & 1,62 & 5,88 & 1,36 & 13,0 \\
\hline \multicolumn{9}{|l|}{ Proteína } \\
\hline LGC 7105 & 21 & $0,17 \pm 0,04^{* *}$ & 5,79 & 2,94 & 0,00 & 0,00 & 1,44 & 18,3 \\
\hline ERM BB $501 \mathrm{~A}$ & 33 & $22,5 \pm 0,14^{*}$ & 1,79 & 0,13 & 0,50 & 2,17 & 0,66 & 6,27 \\
\hline muva-HA-1508 & 24 & $46,6 \pm 0,2$ & 0,77 & 0,34 & 0,10 & 0,21 & 0,34 & 1,66 \\
\hline \multicolumn{9}{|l|}{ Cenizas } \\
\hline LGC 7105 & 21 & $0,19 \pm 0,01$ & 16,62 & 8,26 & 0,03 & 13,63 & 3,30 & 45,7 \\
\hline LGC 7103 & 21 & $1,64 \pm 0,17$ & 2,42 & 1,82 & 0,04 & 2,50 & 0,65 & 9,17 \\
\hline ERM BB $501 \mathrm{~A}$ & 24 & $32,3 \pm 0,4^{a}$ & 2,49 & 0,62 & 0,90 & 2,71 & 0,74 & 6,86 \\
\hline
\end{tabular}

$\mathrm{U}_{\text {rel, }}$ y HORRAT $\mathrm{r}_{\mathrm{r}} \mathrm{DER}_{\mathrm{r}}$ : Desviación estándar relativa de la repetibilidad. $\mathrm{DER}_{\mathrm{R}}$ : Desviación estándar relativa de la reproductibilidad. SA: Sesgo absoluto (valor absoluto). SR: Sesgo relativo. M: Media aritmética \pm límite de confianza (95\%). MCR: Materiales de referencia certificados. n: número de replicados.

** g de nitrógeno/100g. *g de nitrógeno/kg. ${ }^{\mathrm{g}} \mathrm{de}$ cenizas $/ \mathrm{kg}$.

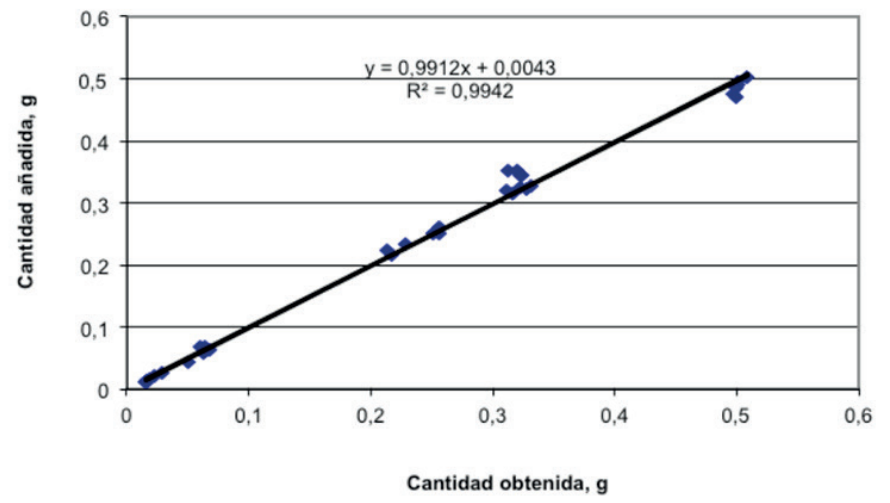

Figura 1. Curva de regresión lineal (grasa medida frente a grasa añadida) en muestras fortificadas. El coeficiente de correlación al cuadrado o de regresión lineal (R2) es igual a 0,9942. El test $F$ de Mandel, $(P G=12,09 ;$ Fcrítico $=2,818 ; P=0,05)$ indica que el rango de trabajo no es igual al rango lineal para una masa de grasa recuperada a una concentración baja y alta.
Tabla 2. Diferencia máxima entre duplicados de muestras de platos cocinados y de raciones de combate.

\begin{tabular}{|cc|}
\hline MACRONUTRIENTES & DIFERENCIA \\
\hline Humedad, $\%$ & $\%$ \\
$40-62$ & 0,65 \\
$62-71$ & 0,60 \\
$71-95$ & 0,50 \\
Grasa, $\%$ & \\
$1,5-3,5$ & 0,25 \\
$3,5-8$ & 0,60 \\
$8-26$ & 1,00 \\
$26-50$ & 2,40 \\
Proteína, \% & \\
$0,5-1$ & 0,09 \\
$1-2$ & 0,15 \\
$2-5$ & 0,25 \\
$5-47$ & 0,5 \\
Cenizas, \% & \\
$0,4-1,5$ & 0,05 \\
$1,5-4$ & 0,08 \\
\hline
\end{tabular}


Pérez Grana R.

Tabla 3. Resultados de análisis de ensayos de aptitud.

\begin{tabular}{|c|c|c|c|c|c|c|c|}
\hline ALIMENTO & NUTRIENTE & $\mathbf{V}_{\mathrm{a}}$ & $\mathbf{V}_{\mathrm{o}}$ & $\mathbf{Z}_{\text {score }}$ & $\mathbf{S}_{\mathrm{R}}$ & $\mathrm{DER}_{\mathrm{R}}, \%$ & HORRAT \\
\hline \multirow[t]{3}{*}{ Paté } & Humedad & 54,06 & 54,18 & 0,16 & 0,16 & 0,34 & 0,28 \\
\hline & Proteína & 11,57 & 12,18 & 1,78 & 0,27 & 2,37 & 0,85 \\
\hline & Grasa & 30,4 & 29,51 & $-1,44$ & 0,54 & 1,78 & 0,74 \\
\hline \multirow[t]{4}{*}{ Queso curado } & Humedad & 71,36 & 28,64 & 0,1 & 0,03 & 0,74 & 0,35 \\
\hline & Proteína & $24,51 *$ & $24,43^{*}$ & $-0,2$ & 1,42 & 2,64 & 1,06 \\
\hline & Grasa & $54,07 *$ & $38,58^{*}$ & $-1,1$ & 0,31 & 1,25 & 0,56 \\
\hline & Cenizas & $4,18^{*}$ & $4,15^{*}$ & $-0,7$ & 0,68 & 0,95 & 0,29 \\
\hline \multirow[t]{4}{*}{ Salmón } & Humedad & 62,11 & 62,3 & 0,4 & 0,05 & 1,22 & 0,56 \\
\hline & Proteína & 17,53 & 17,53 & 0,0 & 0,66 & 4,15 & 1,58 \\
\hline & Grasa & 15,91 & 14,57 & $-1,8$ & 0,32 & 1,85 & 0,69 \\
\hline & Cenizas & 4,21 & 4,17 & $-1,4$ & 0,38 & 0,63 & 0,19 \\
\hline
\end{tabular}

$\mathrm{V}_{\mathrm{a}}$ :Valor asignado: $\mathrm{V}_{\mathrm{o}}$ :Valor medio. $\mathrm{S}_{\mathrm{R}}$ : Reproducibilidad. $\mathrm{DER}_{\mathrm{R}}$ : Desviación estándar relativa de la reproducibilidad.* Valores expresados sobre sustancia seca.

Tabla 4. Media aritmética, valor máximo y mínimo $\pm U_{\text {rel }}$ de humedad, proteína, grasa y cenizas en 51 muestras de purés de dietas geriátricas y composición nutricional en 5 muestras representativas de ellas. *Valores por debajo del rango de determinación.

\begin{tabular}{|lcccc|}
\hline & PURÉS & HUMEDAD & GRASA & \multicolumn{2}{c|}{$\begin{array}{c}\text { PROTEÍNA } \\
\text { \% }\end{array}$} & $\begin{array}{c}\text { CENIZAS } \\
\text { \% }\end{array}$ \\
\hline Media aritmética & $\mathbf{\%}$ & 3,15 & 5,17 & 0,98 \\
SD & 81,3 & 1,27 & 2,14 & 0,48 \\
Valor máximo & 4,0 & $6,06 \pm 1,46$ & $10,9 \pm 1,2$ & $2,45 \pm 0,21$ \\
Valor mínimo & $89,4 \pm 1,5$ & $<3,5(0,84)^{*}$ & $1,11 \pm 0,19$ & $<0,40(0,31)^{*}$ \\
Rango & $72,4 \pm 1,3$ & 5,22 & 9,80 & 2,14 \\
Pollo & 17,0 & $4,81 \pm 1,49$ & $10,9 \pm 1,2$ & $0,98 \pm \pm 0,22$ \\
Verduras & $74,4 \pm 1,2$ & $4,61 \pm 1,44$ & $5,38 \pm 0,74$ & $1,18 \pm 0,27$ \\
Huevo & $80,4 \pm 1,3$ & $4,01 \pm 1,25$ & $4,65 \pm 0,64$ & $0,65 \pm 0,22$ \\
Vegenat pescado & $77,5 \pm 1,3$ & $4,15 \pm 1,29$ & $8,85 \pm 0,95$ & $1,14 \pm 0,26$ \\
Zanahoria con ternera & $72,4 \pm 1,3$ & $<3,5(1,30)^{*}$ & $4,91 \pm 0,68$ & $<0,40(0,31)^{*}$ \\
\hline
\end{tabular}

SD: Desviación estándar.

No hay que olvidar que el muestreo es un factor decisivo para asegurar la representatividad de los resultados y tiene la mayor fuente de variabilidad en la calidad de la medida ${ }^{24}$. Hay autores que opinan que la incertidumbre generada por el muestreo debería ser tomada en consideración ${ }^{25}$. Por otro lado, en el valor obtenido en un proceso analítico, influye el sesgo, en sus diferentes etapas, y el error aleatorio, según la expresión:

$\mathrm{y}=\mu+\beta_{\text {Sesgo muestreo }}+\delta_{\text {Sesgo método }}+\lambda_{\text {Sesgo analista }}+\varphi_{\text {Sesgo instrumento }}+\Delta_{\text {Sesgo dia }+} \varepsilon$.

$\mathrm{Y}$ el error total estima la distribución del error alrededor de un valor de referencia ${ }^{26}$, con la siguiente ecuación: $x_{i}=\mu \pm f(\sigma, \delta)$.

Además, en muchos casos el muestreo hace una sustancial o incluso contribución dominante a la incertidumbre combinada de la medida ${ }^{27-29}$

$\mathrm{u}_{\mathrm{c}}=\sqrt{u_{\text {Muestra }}^{2}+u_{\text {Análisis }}^{2}}$, debido a las fuentes de incertidumbre que afectan al muestreo (heterogeneidad, estado físico de la muestra, transporte y conservación, etc.). A pesar de que la incertidumbre del muestreo contribuye a la incertidumbre combinada, la Guía
EURACHEM/CITAC “Quantifying Uncertainty in Analytical Measurement", trata al muestreo como una fuente de incertidumbre y en la estimación de la $\mathrm{u}_{\mathrm{c}}$, asocia los términos incertidumbre de la precisión y sesgo, sin tener en cuenta la incertidumbre del muestreo. Una metodología de muestreo duplicado, por ejemplo, mediante un diseño experimental balanceado, acoplado con estadística robusta RANOVA, se podría utilizar para determinar la incertidumbre expandida relativa global del proceso de muestreo y del método de análisis ${ }^{30}$. Esta metodología presenta algunos inconvenientes, ya que implica tomar al menos un $10 \%$ de la totalidad de las muestras o $n \geq 8^{31}$. También supone validar un plan de muestreo que confirme que la incertidumbre generada pueda encontrarse con los requerimientos del "fitness for purpose" 32 .

\section{Preparación de la muestra}

El cálculo llevado a cabo para la estimación de la incertidumbre durante la validación, lleva implícito la incertidumbre debida a la 
preparación física de la muestra ${ }^{33}$, que conlleva unas fuentes de incertidumbre significativas (homogeneización/submuestreo, trituración, etc.) y una causa de error importante en el análisis nutricional, incluso superior al muestreo ${ }^{34}$.

\section{Validación de procedimientos analíticosv}

En conservas de raciones de combate y platos cocinados, las posibles matrices pueden ser muy numerosas. Por tanto, se han seleccionado MRC similares a las de las futuras muestras de rutina objeto del análisis ${ }^{35}$.

En la validación de métodos analíticos cuantitativos, típicamente, se determina la precisión, exactitud/sesgo, linealidad, LD, LC, robustez y selectividad. Como los procedimientos de ensayo se aplican al análisis de componentes mayoritarios, los estudios de los parámetros de la calidad, se han centrado en el estudio de la precisión y el sesgo.

La precisión intermedia o reproducibilidad intralaboratorio se halla a partir de la desviación estándar de los replicados. Otra posibilidad es estimar la varianza dentro de días y la varianza entre días, utilizando una tabla ANOVA; así, la varianza de la precisión intermedia, $\mathrm{S}_{\mathrm{I}}^{2}=\mathrm{S}_{\text {serie }}^{2}+\mathrm{S}_{\text {repetibilidad. }}^{2}$

En la estimación de la precisión también se han tenido en cuenta las recomendaciones del International Conference of Harmonization (ICH, 1996). Los valores que se obtienen son aceptables, aunque en cenizas para un valor certificado del $0,22 \%$, fuera del rango de determinación en las muestras reales, se alcanza una $D_{E R}$ del $16,62 \%$, que se considera dentro de los límites del trabajo científico.

El coeficiente de correlación al cuadrado o de regresión lineal $\left(\mathrm{R}^{2}\right)$ es igual a 0,9942 , y es un indicador de correlación, pero no de linealidad. No obstante, se considera un valor dentro de lo esperado, tratándose de muestras fortificadas. Efectivamente, el test $\mathrm{F}$ de Mandel, $\left(\mathrm{PG}=12,09 ; \mathrm{F}_{\text {crítico }}=2,818 ; \mathrm{P}=0,05\right)$ indica que el rango de trabajo no es igual al rango lineal para una masa de grasa recuperada a una concentración baja y alta. En cambio, los resultados son lineales en ensayos con aceite de ricino, obteniéndose recuperaciones entre el $99 \%$ y el $100 \%$.

No se evidencian errores sistemáticos durante la ejecución de los procedimientos analíticos y todos los ensayos de aptitud resultan satisfactorios. Se debe de tener presente que la aplicación de tests estadísticos para comprobar que el procedimiento de ensayo es trazable, lleva consigo el riesgo de cometer un "error $\alpha$ " o un "error $\beta$ ". Así, la probabilidad de cometer un "error $\beta$ " depende de la incertidumbre de los MRC, precisión del método analítico, del valor tipificado verdadero, $\mathrm{z}_{\mathrm{v}}$, $\mathrm{y}$ de otros factores.

La ISO "Guide to the Expression of Uncertainty in Measurement" (1993) recomienda corregir los resultados por el sesgo significativo y no tener en cuenta este sesgo para incrementar la incertidumbre. A pesar de ello, parece razonable no corregir cada resultado individual por el sesgo, y en su lugar incluir el sesgo en la incertidumbre combinada. Se suele adoptar el criterio de corregir los resultados por el sesgo, cuando el error sistemático es estadísticamente significativo, y de abandonar el sesgo y no corregir los futuros resultados cuando el sesgo, $\delta$, no es significativo al compararlo con la incertidumbre combinada, $\delta \leq \mathrm{k} \mathrm{u}(\delta)^{36}$.

En este sentido, existen diferentes expresiones ${ }^{37}$ que se utilizan de forma habitual para la incorporación del sesgo en la estimación de la incertidumbre expandida:
$\mathrm{U}=\mathrm{U}+\delta ; \mathrm{U}(\mathrm{RSSu})=\mathrm{k} \sqrt{u^{2}+\delta^{2}} ; \mathrm{U}(\mathrm{RSSU}) \sqrt{U^{2}+\delta^{2}} ;$ $\mathrm{SUMU}\left[\mathrm{U}_{+}=\mathrm{U}-(-\delta) ; \mathrm{U}_{-}=\mathrm{U}+(-\delta)\right]$.

La incertidumbre se estima conjuntamente considerando el proceso analítico como una sola etapa y, previamente, se identifican las fuentes de incertidumbre.

Por ejemplo, la etapa de hidrólisis en la determinación de grasa es una fuente de incertidumbre significativa. Debe tenerse en cuenta que en operaciones tales como hidrólisis, filtración, extracción, no se garantiza la conservación de la masa total de analito. Por otro lado, se entiende que ignorar el sesgo no significativo podría infraestimar la incertidumbre ${ }^{38}$, especialmente cuando la incertidumbre del sesgo es grande y cuando el sesgo contribuye de forma significativa a la incertidumbre. Por consiguiente, se decide tener en cuenta el sesgo en el cálculo de la incertidumbre. También se recomienda la inclusión del sesgo no significativo en la incertidumbre expandida, cuando la incertidumbre del sesgo $\mathrm{u}(\delta)$ es mayor que el $30 \%$ de la incertidumbre combinada ${ }^{39}$.

Otro aspecto a considerar en la validación es el número de replicados, que deben ser suficientes para proporcionar una estimación fiable de la desviación estándar. La citada ISO 5725-3 recomienda realizar al menos 15 replicados, aunque el criterio más común es realizar 10 réplicas. En nuestro caso la muestra de referencia se analiza más de 10 veces, a los efectos de disminuir tanto el "error $\alpha$ " como el "error $\beta$ ", y de aumentar la potencia del test (1- $\beta)$.

Mediante la expresión siguiente adaptada a un laboratorio individual, se puede determinar el número de veces que debe analizarse la muestra para detectar un sesgo mínimo ${ }^{40}$ :

$\lambda \geq\left(\mathrm{t}_{\alpha / 2}+\mathrm{t}_{\beta}\right) \sqrt{u_{\text {ref }}^{2}+\frac{S_{i}^{2}}{n}}$. La única incógnita es $n$, ya que debe

conocerse la precisión intermedia del método y la incertidumbre del valor de referencia, una vez fijado un sesgo mínimo y la probabilidad $\alpha$ y $ß$. También son útiles otras expresiones que nos permiten determinar el límite de detección del sesgo, $\Delta_{\mathrm{Db}}$, y el valor crítico,

$\Delta_{\mathrm{c}}: \Delta_{\mathrm{Db}}=\left(\mathrm{t}_{1-\alpha / 2}+\mathrm{t}_{1-\beta}\right) \sigma / \sqrt{n}+2 \mathrm{U} ; \Delta_{\mathrm{c}}=\left(\mathrm{t}_{1-\alpha / 2}\right) \sigma / \sqrt{n}+\mathrm{U}$, conociendo el número de replicados. El test para el sesgo se hace al nivel de significación $\alpha=0,05$, para detectar un diferencia absoluta, $\Delta_{\mathrm{b}}$, entre la media de un mensurando y el valor atribuido al mensurando ( $\beta=$ riesgo de falso negativo), y $1-\beta=0,95$, potencia del test). Por ejemplo, para proteínas obtenemos a un nivel de concentración bajo $\left(\Delta_{\mathrm{Db}}=0,05 \% ; \Delta_{\mathrm{c}}=0,02 \%\right)$; a un nivel de concentración medio $\left(\Delta_{\mathrm{Db}}=0,17 \% ; \Delta_{\mathrm{c}}=0,08 \%\right)$ y a un nivel de concentración alto $\left(\Delta_{\mathrm{Db}}=\right.$ $\left.0,62 \% ; \Delta_{\mathrm{c}}=0,32 \%\right)$.

No se han hallado resultados discrepantes mediante el test de Grubb's $\alpha=0,05$, en ninguno de los procedimientos analíticos sometidos a validación. Basándonos en el teorema del límite central, nos ha permitido aplicar inferencias de estadística paramétrica a los resultados obtenidos de medidas repetidas.

Los ensayos de aptitud de macronutrientes constituyen una herramienta indispensable en la validación de los métodos analíticos ${ }^{41-43}$. El valor HORRAT, definido como la relación entre la desviación estándar de la reproducibilidad y la función de Horwitz $\left(\mathrm{s}_{\mathrm{R}} / \mathrm{H}\right)$, muestra que los valores hallados se encuentran en el intervalo $0,5<\mathrm{Hs}_{\mathrm{R}}<2.0^{44}$, lo que indica que los procedimientos de ensayo tienen una veracidad satisfactoria. Similares resultados se obtienen en el cálculo de $\operatorname{HORRAT}_{r}$, en condiciones de reproductibilidad intralaboratorio. Es 


\section{R. Pérez Grana}

de destacar que para una concentración certificada de humedad de $71,3 \mathrm{~g} / 100 \mathrm{~g}$, se obtiene un valor $\mathrm{HORRAT}_{\mathrm{r}}$ de 0,15 , con una $\mathrm{U}_{\text {rel }}$ del $1,67 \%$, y con un valor medio que cae dentro del intervalo certificado. Asimismo, en cenizas a la concentración de $0,19 \mathrm{~g} / 100 \mathrm{~g}$, se halla un valor HORRAT $\mathrm{H}_{\mathrm{r}}$ e 3,3, que en absoluto invalida el método analítico.

\section{Estimación de la incertidumbre}

Es posible estimar la $U_{\text {rel }}$ combinando otros términos de incertidumbre estándar, siguiendo la ley de propagación de errores, con las expresiones 1, 2 y 3 que se indican abajo. Por ejemplo, a través de ensayos realizados de forma paralela en la estimación de la incertidumbre de la humedad, con un MRC de concentración $61,8 \mathrm{~g} / 100 \mathrm{~g}$, se halla con la expresión 1) una desviación $(-0,04 \%)$, respecto a la $\mathrm{U}_{\text {rel, }}$ declarada en la tabla 1.

1) $\left.\left.\sqrt{\mathrm{u}_{\text {precisión }}^{2}+\mathrm{u}_{\text {trazab. }}^{2}+\mathrm{u}_{\text {matriz }}^{2}} ; 2\right) \sqrt{\mathrm{u}_{\text {MRC }}^{2}+\mathrm{u}_{\text {método }}^{2}+\mathrm{u}_{\text {muestra }}^{2}} ; 3\right) \sqrt{\mathrm{u}_{\text {sesgo }}^{2}+\mathrm{u}_{\text {duplicados }}^{2}}$ $\mathrm{u}_{\text {precisión }}=\frac{\frac{s_{I}}{\sqrt{n}}}{x} ; \mathrm{u}_{\text {trazabilidad }}=\frac{\sqrt{u_{r e f}^{2}+\left(\frac{s_{I}}{\sqrt{n}}\right)^{2}}}{x_{M R C}}$

De donde, $x$ es el valor medio; $\mathrm{x}_{\mathrm{MRC}}$ el valor certificado; $\mathrm{S}_{\mathrm{I}}$ la precisión intermedia; $\mathrm{u}_{\mathrm{ref}}$, la incertidumbre estándar del MRC.

El ensayo de homogeneidad de Bartlett demuestra que las varianzas de la repetibilidad de humedad, en las matrices ensayadas (melocotón en almíbar, paté a la pimienta y atún en aceite) son homocedásticas $(\alpha=0,05 ; n=5)>M_{\text {cal }}$. Se ha utilizado este test por considerarlo como el más adecuado dada la normalidad de las series.

El término, $\mathrm{u}_{\text {matriz }}=\left(S_{\text {conjunta }}-\frac{S_{r}}{x_{D}}\right)$; siendo $\mathrm{x}_{\mathrm{D}}$, el valor medio de los duplicados, $\mathrm{y}, \mathrm{S}_{\mathrm{r}}$ la desviación estándar calculada en condiciones de repetibilidad, según la expresión:

$$
\mathrm{S}_{\mathrm{r}}=\sqrt{\frac{S_{1}^{2}+S_{2}^{2}+S_{3}^{2}+\cdots S_{n}^{2}}{n}}
$$

$$
\text { Y el término, } \mathrm{S}_{\text {conjunta }}=\sqrt{\frac{\left(n_{1}-1\right) x D E R_{1}^{2}\left(n_{2}-1\right) x D E R_{2}^{2}+\cdots\left(n_{n-1}\right) x D E R_{n}^{2}}{\left(n_{1}-1\right)+\left(n_{2}-1\right)+\cdots+\left(n_{n}-n\right)}}
$$

De dónde, DER, es la desviación estándar relativa de los replicados en condiciones de repetibilidad $\mathrm{y}, n$, el número de replicados.

$\mathrm{La}_{\text {rel, }}$ que afecta a la matriz, en las condiciones del ensayo de humedad, se considera despreciable. Igualmente, se demuestra en ensayos con diversas matrices de conservas de raciones de combate (paté a la pimienta, calamares [Loligo vulgaris Lamarck, 1798] en su tinta, ensalada campera, caballa [Scomber scombrus Linnaeus, 1758] en aceite, carne de vacuno con guisantes, etc.) que la incertidumbre de la matriz resulta también despreciable en el caso de la proteína y cenizas.

Tal y como se puede apreciar en las expresiones 2) y 3), tienen en cuenta la variabilidad de las muestras reales. Según se ha demostrado en matrices similares a las utilizadas en ensayos de repetibilidad para la estimación de la $\mathrm{u}_{\text {matriz }}$ en raciones de combate, la variabilidad natural podría sobreestimar la $\mathrm{U}_{\text {rel. }}$. Por consiguiente, se decide no incluir el término $\mathrm{u}_{\text {muestra }} \mathrm{u}_{\text {duplicados }}$ de las muestras reales o de rutina en la estimación de la $\mathrm{U}_{\text {rel }}$.

\section{Contenido en macronutrientes}

Los resultados medios de macronutrientes hallados en las muestras de purés, son similares a los que figuran en las tablas de composición de alimentos para marcas comerciales ${ }^{45}$. Por ejemplo, puré de pollo con verduras (humedad, 77\%; proteína, 5,6\%; grasa, $4,1 \%$ ), crema de espárragos (humedad, $83 \%$; proteína, 1,76\%; grasa, $3,52 \%$ ), puré de ternera a la jardinera (humedad, 76\%; proteína, $5 \%$; grasa, $3,9 \%$ ). Y a los datos obtenidos en nuestro laboratorio a partir del análisis de marcas comerciales: puré de pollo con verduras (humedad, 76,39\%, grasa, 2,65\%, proteínas, 5,48\%; puré de merluza con bechamel (humedad, 78,48\%; grasa, 2,54 \%; proteínas, $4,87 \%$. Es de destacar que en las 51 muestras analizadas, los resultados ofrecen una notable variabilidad, lo que depende de los ingredientes de la receta (pollo, verduras, ternera, pescado, legumbres, vegetales, etc.) y del porcentaje de agua.

\section{CONCLUSIONES}

Los parámetros de la calidad estudiados durante la validación y los resultados de los ensayos interlaboratorio, demuestran que los procedimientos analíticos son útiles para la finalidad perseguida.

Los resultados analíticos de los purés, permiten conocer la composición exacta en los macronutrientes ensayados, lo que puede resultar de utilidad para valorar la ingesta en este grupo de población.

La composición nutricional de los purés depende, en gran medida, del contenido de agua, y algunos son pobres en los macronutrientes estudiados. El rango de valores máximo y mínimo, proporcionalmente el más elevado, corresponde a las proteínas. El alcance de la acreditación concedida por (ENAC, España) afecta a humedad (40-95\%); proteína $(0,5-46 \%)$, grasa $(3,5-50 \%)$ y cenizas $(0,4-4 \%)$.

\section{BIBLIOGRAFÍA}

1. Milà Villarroel R, Abellana Sangrà R, Farran Codina A. Valoración nutricional de los menús en cinco residencias geriátricas. Adecuación a las ingestas recomendadas para la población anciana. Actividad dietética, 2009; 13 (2):51-56.

2. Ley 17/2011, de 5 de julio, de Seguridad Alimentaria y Nutrición.

3. Martín Salinas C, Martínez Montero P. Alimentación en la edad avanzada. En: Martín Salinas C, Motilla Valeriano T, Díaz Gómez J, Martínez Montero P. Nutrición y dietética. Madrid: DAE, S.L. 2000:281-295.

4. Muñiz Muñoz C, López Nomdedeu C, Sendra Pina P. Alimentación en las personas mayores. En: Vázquez C, De Cos AI, López Nomdedeu C. Alimentación y nutrición. Madrid: Díaz de Santos, 2005: 227-237.

5. Gómez Enterría P, Laborda González L. Dieta en las distintas etapas de la vida. En: De Luís Román DA, Bellido Guerrero D, García Luna PP. Dietoterapia, nutrición clínica y metabolismo. Madrid: Díaz de Santos, 2010: 601-611.

6. Pérez Grana, R. Exactitud de las tablas de composición de alimentos en la determinación de nutrientes. Sanidad Militar 2013; 69(2): 102-111.

7. ISO Guide 8402: Quality-Vocabulary. Genève, 1994.

8. UNE-EN ISO/IEC 17025. Requisitos generales relativos a la competencia de los laboratorios de ensayo y calibración. AENOR, Madrid. 2000.

9. UNE-ISO 3534-1. Vocabulario y símbolos. Parte 1: Términos estadísticos generales y términos empleados en el cálculo de probabilidades. 2008.

10. BIPM, CEI, IFCC, ILAC, ISO, UICPA, UIPPA, OIML. Evaluation of measurement data-Guide to the expression of uncertainty in measurement. First Edition 2008.

11. International Vocabulary of Metrology. Basic and general concept and associated term (VIM) $3^{\text {rd }} 2008$ JCGM 2012.

12. Thompson M, Fearn T. What exactly is Fitness for Purpose in Analytical Measurement? Analyst 1996; 121:275-278.

13. ISO 1442-1997. Meat and meat products. Determination of moisture.

14. ISO 937-1978. Meat and meat products. Determination of nitrogen content.

15. ISO 1443- 1973. Meat and meat products. Determination of total fat.

16. ISO 936-1998. Meat and meat products. Determination of total ash 


\section{Validación y acreditación de procedimientos analíticos de macronutrientes: aplicación al análisis...}

17. McNeal JE. Meat and Meat Products. En: Official Methods of Analysis. Volume two. AOAC. $15^{\text {th }}$ Edition. Arlington. USA: 1990: 931-948.

18. Lane RH. Cereal Foods. En: Official Methods of Analysis. Volume two. AOAC $15^{\text {th }}$ Edition. Arlington. USA: 1990: 777-801.

19. Clarke MA. Sugars and sugar products. En: Official Methods of Analysis. Volume two. AOAC. $15^{\text {th }}$ Edition. Arlington. USA: 1990: 1010-1044.

20. Durden D. Evaluation of the Order of Hierarchical Structures for the Calculation of Method Uncertainty Using Nested and Other Designs. J AOAC International, 2011; 94:1643-1649.

21. ISO 5725-3. Accuracy (trueness and precision) of measurement methods and results. Intermediate measures of the precision of a standard measurement. ISO, Genève, 1994.

22. ISO 5725-6. Accuracy (trueness and precision) of measurement methods and results. Use in practice of accuracy values. ISO, Genève, 1994.

23. Rios A, Valcárcel M. A view of uncertainty at the bench analytical level. Accred Qual Assur 1998; 3:14-19.

24. Williams JSE. Considerations of comparability and traceability for food analysis. Accred Qual Assur 2004; 9: 557-560.

25. Hund E, Massart LD, Smeyers-Verbeke J. Operational definitions of uncertainty. Trends Anal Chem 2001; 20:394-406.

26. Rozet E, Marini RD, Ziemons E, Dewé W, Rudaz S, Boulanger B, Hubert PH. Total error and uncertainty: Friends or foes? Trends Anal Chem 2011; 30:797-806.

27. Analytical Methods Committee. Measurement uncertainty arising from sampling: the new Eurachem Guide, 2008. ISSN 1757-5958.

28. Ramsey MH, Ellison SLR. Measurement uncertainty arising from sampling: A guide to methods and approaches. EURACHEM/CITAC, 2007.

29. Galbán J, Ubide C. Uncertainty due to the quantification step in analytical methods. Talanta 2007; 71:1339-1344.

30. Rostron PD, Ramsey MH. Coste effective robuste estimation of measurement uncertainty from sampling using unbalanced ANOVA. Accred Qual Assur 2012; 17: 7-14.

31. Lyn JA, Ramsey MH, Coad S, Damant P, Word R, Boon KA. The duplicate method of uncertainty estimation: are eight targets enough? Analyst 2007; 132: $1147-1152$.
32. Ramsey MH, Thompson M. Uncertainty from sampling in the context of fitness for purpose. Accred Qual Assur 2007; 12: 503-513.

33. Lyn JA, Ramsey MH, Fussell RJ, Wood R. Measurement uncertainty from physical sample preparation: estimation including systematic error. Analyst 2003; 128:1391-1398.

34. Ellison SLR, Hardcastle WA. Causes of error in analytical chemistry results of web-based survey of proficiency testing participants. Accred Qual Assur, 2012; 17:453-464.

35. Thompson M, Ellison SLR, Wood R.A. Harmonised Guidelines for single-laboratory validation of methods of analysis. Pure Appl Chem 2002; 74 (5): 835-855.

36. Haesserlbarth W. Accounting for bias in measurement uncertainty estimation. Accred Qual Assur 2004; 9: 509-514

37. Synek V. Attempts to include uncorrected bias in the measurement uncertainty Talanta 2005: 65:829-837.

38. Linsinger TPJ. Use of recovery and bias information in analytical chemistry and estimation of its uncertainty contribution. Trends Anal Chem 2008; 27:916-923.

39. Maroto A, Boqué R, Riu J, Rius X. Should non-significant bias included in the uncertainty budget?. Accred Qual Assur 2002; 7:90-94.

40. Kuttatharmmakul S, Massart DL, Smeyers-Verbeke J. Comparison of alternative measurement methods. Anal Chim Acta 1999; 391:203-225.

41. Sykes M. Proficiency testing for the improvement of analytical practice. Accred Qual Assur 2012: Discussion Forum.

42. Thompson M, Ellison SLR, Wood R. The international harmonized protoco for the proficiency testing of analytical chemistry laboratories. Pure Appl Chem 2006; 1:145-196.

43. Chung SWC, Tran JCH, Wong WW. Capability of laboratories to determine core nutrients in foods: results in an international proficiency test. Accred Qual Assur 2012; 17: 35-34.

44. Thompson M, Guffogg S, Stangroom S, Osborne P, Key P, Wood R. Precision estimates produced by specially-designed ruggedness tests compared with those derived from collaborative trials, in relation to estimation of measurement uncertainty. Analyst 2002; 127: 1669-1675.

45. Mataix Verdú, J. Tablas de composición de alimentos. Instituto de nutrición y tecno-

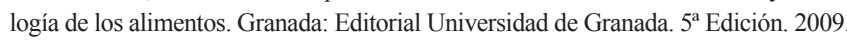

